\title{
La Mediación como solución de conflictos en el sistema escolar de la unidad educativa Babahoyo
}

\section{Mediation as a conflict resolution in the school system of the Babahoyo educational unit}

\author{
Patty Del Pozo-Franco \\ pattydelpozofranco@gmail.com \\ Universidad Regional Autónoma de Los Andes, Babahoyo \\ Ecuador \\ https://orcid.org/0000-0002-2479-092X \\ Kevin Marcos Lynch-Becilla \\ lynchbecilla83@gmail.com \\ Universidad Regional Autónoma de Los Andes, Babahoyo \\ Ecuador
}

Recibido: 31 de octubre de 2020

Revisado: 10 de octubre de 2020

Aprobado: 05 de diciembre de 2020

Publicado: 10 de diciembre de 2020 
lustitia Socialis. Revista Arbitrada de Ciencias Jurídicas.

Año V. Vol. V. №3. Edición Especial. 2020-III:

Universidad Regional Autónoma de los Andes

Hecho el depósito de Ley: FA2016000064

ISSN: 2542-3371

FUNDACIÓN KOINONIA (F.K). Santa Ana de Coro, Venezuela

Patty Del Pozo-Franco; Kevin Marcos Lynch-Becilla

\title{
RESUMEN
}

La investigación tiene como objetivo analizar la eficacia de la Ley de Arbitraje y Mediación, en el sistema escolar de Unidad Educativa Babahoyo(UEB). Se desarrolló bajo el enfoque cuantitativo de tipo descriptiva, de campo no experimental, teniendo como población los miembros de la comunidad educativa de Babahoyo, se aplicó el procedimiento de muestreo aleatorio esterificado por afijación proporcional obteniendo lo siguiente: estudiantes 494, padres de familia 466, docentes 22 y administrativo 2 , constituyendo así la muestra, a los cuales se procedió a aplicar la encuesta conformada por un cuestionario de 10 preguntas, se eligió como técnica el análisis documental y la encuesta. Se sugiere que, para la promoción de un ambiente favorable en la unidad educativa, se bebe divulgar una adecuada interpretación del alcance del Artículo 52 de la Ley de Arbitraje y Mediación, así como promover su modificación y creación de un centro de mediación.

Descriptores: Solución de conflictos; mediación; ambiente educacional. (Palabras tomadas del Tesauro UNESCO).

\begin{abstract}
The research aims to analyze the effectiveness of the Arbitration and Mediation Law in the school system of the Babahoyo Educational Unit (UEB). It was developed under a descriptive quantitative approach, non-experimental field, having as population the members of the educational community of Babahoyo, the procedure of random sampling esterified by proportional allocation was applied obtaining the following: students 494, parents 466, teachers 22 and administrative 2, thus constituting the sample, to which the survey consisting of a questionnaire of 10 questions was applied, the documentary analysis and the survey were chosen as the technique. It is suggested that, in order to promote a favorable environment in the educational unit, an adequate interpretation of the scope of Article 52 of the Arbitration and Mediation Law should be disclosed, as well as promoting its modification and creation of a mediation center.
\end{abstract}

Descriptors: Conflict resolution; mediation; educational environment. (Words taken from the UNESCO Thesaurus). 
Iustitia Socialis. Revista Arbitrada de Ciencias Jurídicas.

Año V. Vol. V. №3. Edición Especial. 2020-III:

Universidad Regional Autónoma de los Andes

Hecho el depósito de Ley: FA2016000064

ISSN: 2542-3371

FUNDACIÓN KOINONIA (F.K). Santa Ana de Coro, Venezuela

Patty Del Pozo-Franco; Kevin Marcos Lynch-Becilla

\section{INTRODUCCIÓN}

Los conflictos surgen ante la oposición de intereses o posiciones divergentes entre dos o más personas, de tal modo que se ven involucradas emotiva y sentimentalmente en la situación. Los conflictos son inherentes a nosotros y nosotras como seres gregarios. Pueden resultar en relaciones fortalecidas e individuos con mejores herramientas para gestionar la adversidad, las diferencias y, en general, la habilidad para convivir. (UNICEF, 2019). Así mismo, nos mencionan que presenta las siguientes características: Son situaciones inevitables, propias de la convivencia, que para su resolución requieren la toma de decisiones. En el conflicto no se presenta, necesariamente, la intención de dañar o maltratar al otro. Pueden derivar en el fortalecimiento de las relaciones entre las personas involucradas e incluso, de la convivencia en general. Puede ocurrir entre personas adultas, niños, niñas y adolescentes y entre individuos de estos grupos, con o sin asimetría de poder. Entran en juego las habilidades para el manejo de las emociones, sentimientos e intereses, tanto suyas como de las otras partes del conflicto. Puede dar lugar al desarrollo y profundización de la convivencia. Por ello, en medio de la civilización e intento de convivencia pacífica se examinan opciones que favorezcan la conciliación de las partes involucradas en la polémica; entre las cuales se encuentran los métodos alternativos de resolución de conflictos: la mediación y el arbitraje. (Corvoda-Mendoz, Ochoa-Espinoza \& Durán-Ocampo, 2019)

De lo anterior se resalta que la violencia requiere de prevención; las reglas, normas y leyes, crean una cultura que establece un límite. Así la Constitución, da mucha importancia a la actuación del ciudadano para hacer conciencia y compromiso con el principio de responsabilidad, recogido en el campo del derecho bajo el precepto de "honeste vivire" (vive honestamente), y por ello le permite mediar. El desarrollo de las sociedades cambió el comportamiento de la juventud, cada vez más violenta; que resulta en una deplorable educación, en detrimento de la sociedad.

Igualmente, la aplicación de la ley está pendiente para el caso conflictivo, pero su papel central se pierde, por la necesidad de Deslegalización frente a la solución presentada por el mediador y garantizada por estar in curso frente a los derechos 
particulares reclamados por las partes y que no afectan a la aplicación de la ley por parte del Estado, por no constituir materia de arbitraje fuera de la ley.

De acuerdo al artículo 97.- Todas las organizaciones podrán desarrollar formas alternativas de mediación y solución de conflictos, en los casos que permita la ley; actuar por delegación de la autoridad competente, con asunción de la debida responsabilidad compartida con esta autoridad; demandar la reparación de daños ocasionados por entes públicos o privados; formular propuestas y reivindicaciones económicas, políticas, ambientales, sociales y culturales; y las demás iniciativas que contribuyan al buen vivir.(Asamblea Constituyente, 2008), así mismo el artículo 190, Se reconoce el arbitraje, la mediación y otros procedimientos alternativos para la solución de conflictos. Estos procedimientos se aplicarán con sujeción a la ley, en materias en las que por su naturaleza se pueda transigir (Ibídem). Se pude observar las garantías que brinda el Estado ecuatoriano, en cuanto a la mediación y solución de conflictos.

Continuando con la revisión de las normas en esta materia, según la Ley de Arbitraje y Mediación (2018), en su artículo 43 define la mediación es un procedimiento de solución de conflictos por el cual las partes, asistidas por un tercero neutral llamado mediador, procuran un acuerdo voluntario, que verse sobre materia transigible, de carácter extrajudicial y definitivo, que ponga fin al conflicto.

En este sentido, en la Unidad Educativa Babahoyo (UEB), basados en la aprobación del proyecto: Por una Convivencia Armónica y Solidaria, el Ministerio de Educación le faculta para "la resolución pacífica de conflictos en todos los espacios de la vida personal, escolar, familiar y social”, según el artículo 2 Acuerdo Min. Nº434-12.

Por otro lado, la implementación de la Oficina de Coordinación de Mediación de Conflictos, enmarcado en lo que estipula el artículo 52 de la citada Ley de Arbitraje y Mediación, determina que: las instituciones sin fines de lucro, podrán organizar centros de mediación.

Sin embargo, los planteles carecen de centros de mediación escolar y en La Junta Distrital de Resolución de Conflictos, se incurre en una lentitud burocrática. En contradicción a los que plantea el Ministerio de Educación, "el proceso disciplinario de 
lustitia Socialis. Revista Arbitrada de Ciencias Jurídicas.

Año V. Vol. V. №3. Edición Especial. 2020-III:

Universidad Regional Autónoma de los Andes

Hecho el depósito de Ley: FA2016000064

ISSN: 2542-3371

FUNDACIÓN KOINONIA (F.K). Santa Ana de Coro, Venezuela

Patty Del Pozo-Franco; Kevin Marcos Lynch-Becilla

las faltas muy graves debe ser sustanciado al interior del establecimiento educativo, y las acciones educativas disciplinarias deben ser aplicadas por la Junta Distrital de Resolución de Conflictos". Esto, induce a acciones jurídicas basadas en el papel sancionador de la ley, perdiéndose el papel preventivo de la mediación; en contraposición al mencionado artículo 52 de la Ley de Arbitraje y Mediación, lo que vulnera derechos de los estudiantes, provocando afectación a su desenvolvimiento académico, social y su formación integral. En este sentido el Código Orgánico de la Niñez y adolescencia en su artículo 294 es tácito: La mediación procederá en todas las materias transigibles siempre que no vulneren derechos irrenunciables de la niñez y la adolescencia. Se plantea la siguiente interrogante ¿Cómo influye la solución de conflictos escolares en la eficacia de la Ley de Arbitraje y Mediación, del sistema escolar de la UEB año 2019-2020?

De acuerdo a lo señalado el presente trabajo de revisión, tiene como objetivo analizar la eficacia de la Ley de Arbitraje y Mediación, en el sistema escolar de UEB.

\section{METODOLOGÍA}

El presente estudio se desarrolló bajo el enfoque cuantitativo de tipo descriptiva de campo no experimental, teniendo como población los miembros de la comunidad educativa de Babahoyo (UEB): estudiantes 2646, padres de familia 2500, docentes 116 y administrativos 2 y para la obtención de la muestra se aplicó el procedimiento de muestreo aleatorio esterificado por afijación proporcional obteniendo los siguientes: estudiantes 494, padres de familia 466, docentes 22 y administrativo 2, a los cuales se procedió a aplicar la encuesta conformada por un cuestionario de 10 preguntas, el cual fue validado por el juicio de expertos, calculándose el coeficiente Alfa de Cronbach en razón de prueba piloto, cuyo resultado fue de 0,88 siendo considerado confiable para su aplicación, en tal sentido, los datos compilados fueron procesados a través de la estadística descriptiva. Se eligió como técnica el análisis documental y la encuesta cuyos resultados obtenidos permitieron establecer las conclusiones sobre el tema y de acuerdo a lo plateado por Riofrio (2015). 
lustitia Socialis. Revista Arbitrada de Ciencias Jurídicas.

Año V. Vol. V. №3. Edición Especial. 2020-III:

Universidad Regional Autónoma de los Andes

Hecho el depósito de Ley: FA2016000064

ISSN: 2542-3371

FUNDACIÓN KOINONIA (F.K). Santa Ana de Coro, Venezuela

Patty Del Pozo-Franco; Kevin Marcos Lynch-Becilla

\section{RESULTADOS}

A continuación, se muestran los resultados obtenidos de la encuesta, de acuerdo las preguntas formuladas.

Tabla 1.

Tabulación encuesta a docentes.

\begin{tabular}{|c|c|c|c|c|c|c|}
\hline $\mathbf{N}^{\circ}$ & ITEM & SI\% & NO\% & NULOS & $\begin{array}{c}\text { TOTAL } \\
\text { RESP. }\end{array}$ & $\%$ \\
\hline 1 & $\begin{array}{l}\text { ¿Los estudiantes deben cumplir trabajo } \\
\text { comunitario, debido a los conflictos } \\
\text { escolares? }\end{array}$ & 90,5 & 9,5 & 4,5 & 21 & 95,5 \\
\hline 2 & $\begin{array}{l}\text { ¿Cree usted que algunos estudiantes } \\
\text { sancionados son reincidentes en violencia, } \\
\text { debido a que están resentidos al ser } \\
\text { sancionados por la ley? }\end{array}$ & 50 & 50 & 0 & 22 & 100 \\
\hline 3 & $\begin{array}{l}\text { ¿Cree usted que la lista de casos de violencia } \\
\text { por chismes en redes sociales, es debido a que } \\
\text { no se logra el Buen vivir? }\end{array}$ & $\mathbf{9 0 , 9}$ & 9,1 & 0 & 22 & 100 \\
\hline 4 & $\begin{array}{l}\text { ¿Considera usted que el informe de llamadas } \\
\text { para la intervención de la POLICÍA, } \\
\text { DINAPEN y GOE a la salida de los } \\
\text { estudiantes, es debido a los diferentes tipos de } \\
\text { conflictos que se observan? }\end{array}$ & $\mathbf{9 0 , 9}$ & 9,1 & 0 & 22 & 100 \\
\hline 5 & $\begin{array}{l}\text { ¿El reporte de inspectores sobre los extraños } \\
\text { en el plantel, se debe al aumento de la } \\
\text { violencia? }\end{array}$ & 76,2 & 23,8 & 4,5 & 21 & 95,5 \\
\hline 6 & $\begin{array}{l}\text { ¿Cree usted que la asistencia a talleres de } \\
\text { prevención de la violencia organizados por el } \\
\text { plantel, permite al personal de la UEB ayudar } \\
\text { en la mediación? }\end{array}$ & 100 & 0 & 0 & 22 & 100 \\
\hline 7 & $\begin{array}{l}\text { ¿Considera que la disminución en los datos } \\
\text { estadísticos de estudiantes sancionados, se } \\
\text { debe a la prevención del maltrato escolar? }\end{array}$ & 81,8 & 18,2 & 0 & 22 & 100 \\
\hline 8 & $\begin{array}{l}\text { ¿Existen muchas unidades educativas que no } \\
\text { tienen Centro de Mediación y solución de } \\
\text { conflictos, debido a que la ley no obliga a } \\
\text { crearlas? }\end{array}$ & 54,5 & 45,5 & 0 & 22 & 100 \\
\hline 9 & $\begin{array}{l}\text { ¿Conoce si hay una lista de docentes que } \\
\text { forman comisiones de control, como producto } \\
\text { de la organización de acciones que previenen } \\
\text { o disminuyen el índice de violencia? }\end{array}$ & 61,9 & 38,1 & 4,5 & 21 & 95,5 \\
\hline 10 & $\begin{array}{l}\text { ¿Considera usted que las autoridades deben } \\
\text { gestionar con un proyecto la creación } \\
\text { obligatoria de un centro de mediación y } \\
\text { solución de conflictos, debido a la necesidad } \\
\text { de prevenirlos? }\end{array}$ & 86,4 & 13,6 & 0 & 22 & 100 \\
\hline & TOTAL & $78 \%$ & $21,1 \%$ & $0,9 \%$ & 21,7 & $100 \%$ \\
\hline
\end{tabular}

Fuente: Resultados obtenidos del instrumento aplicado a la comunidad educativa Babahoyo. Autores (2020). 
lustitia Socialis. Revista Arbitrada de Ciencias Jurídicas.

Año V. Vol. V. №3. Edición Especial. 2020-III:

Universidad Regional Autónoma de los Andes

Hecho el depósito de Ley: FA2016000064

ISSN: 2542-3371

FUNDACIÓN KOINONIA (F.K). Santa Ana de Coro, Venezuela

Patty Del Pozo-Franco; Kevin Marcos Lynch-Becilla

Tabla 2.

Tabulación de encuestas a padres de familia.

\begin{tabular}{|c|c|c|c|c|c|c|}
\hline $\mathbf{N}^{\circ}$ & ITEM & SI\% & NO\% & NULOS & $\begin{array}{l}\text { TOTAL } \\
\text { RESP. }\end{array}$ & $\%$ \\
\hline 1 & $\begin{array}{l}\text { ¿Los estudiantes deben aprender a } \\
\text { prevenir la violencia, para disminuir } \\
\text { conflictos escolares? }\end{array}$ & 98,7 & 1,3 & 3,9 & 458 & $96,1 \%$ \\
\hline 2 & $\begin{array}{l}\text { ¿En la actualidad algunos } \\
\text { estudiantes sancionados son } \\
\text { reincidentes en violencia, debido a } \\
\text { que están resentidos al ser } \\
\text { sancionados por la ley? }\end{array}$ & 71,8 & 28,2 & 6,1 & 448 & $93,9 \%$ \\
\hline 3 & $\begin{array}{l}\text { ¿Cree usted que los casos de } \\
\text { violencia por chismes en redes } \\
\text { sociales, es debido a que no se logra } \\
\text { el Buen vivir? }\end{array}$ & 80,8 & 19,2 & 0 & 477 & $100 \%$ \\
\hline 4 & $\begin{array}{l}\text { ¿Sabía usted cree que se llama a la } \\
\text { POLICÍA, DINAPEN y GOE a la } \\
\text { salida de los estudiantes, es debido } \\
\text { a los diferentes tipos de conflictos } \\
\text { que se observan? }\end{array}$ & 77,7 & 22,3 & 3,9 & 458 & $96,1 \%$ \\
\hline 5 & $\begin{array}{l}\text { ¿El reporte de inspectores sobre los } \\
\text { extraños en el plantel, se debe al } \\
\text { aumento de la violencia? }\end{array}$ & 84,6 & 15,4 & 4,8 & 454 & 95,2 \\
\hline 6 & $\begin{array}{l}\text { ¿Cree usted que la asistencia a } \\
\text { talleres de prevención de la violencia } \\
\text { organizados por el plantel, permite al } \\
\text { personal de la UEB ayudar en la } \\
\text { mediación? }\end{array}$ & 96,9 & 3,1 & 4,1 & 457 & 95,9 \\
\hline 7 & $\begin{array}{l}\text { ¿Considera que la disminución de } \\
\text { estudiantes sancionados, se debe a } \\
\text { la prevención del maltrato escolar? }\end{array}$ & 73,4 & 26,6 & 4,6 & 455 & 95,4 \\
\hline 8 & $\begin{array}{l}\text { ¿Sabía usted que existen muchas } \\
\text { unidades educativas que no tienen } \\
\text { Centro de Mediación y solución de } \\
\text { conflictos, debido a que la ley no } \\
\text { obliga a crearlas? }\end{array}$ & 50,8 & 49,2 & 4,1 & 457 & 95,9 \\
\hline 9 & $\begin{array}{l}\text { ¿Por los docentes que forman } \\
\text { comisiones de control, ha } \\
\text { disminuido el índice de violencia? }\end{array}$ & 82,1 & 17,9 & 5,2 & 452 & 94,8 \\
\hline 10 & $\begin{array}{l}\text { ¿Las autoridades deben gestionar } \\
\text { un proyecto de creación obligatoria } \\
\text { de un centro de mediación y } \\
\text { solución de conflictos, debido a la } \\
\text { necesidad de prevenir antes que } \\
\text { sancionar? }\end{array}$ & 97,6 & 2,4 & 4,1 & 457 & 95,9 \\
\hline & TOTAL & $81,4 \%$ & $18,5 \%$ & $4 \%$ & 457,3 & $100 \%$ \\
\hline
\end{tabular}

Fuente: Resultados obtenidos del instrumento aplicado a la comunidad educativa Babahoyo. Autores (2020). 
lustitia Socialis. Revista Arbitrada de Ciencias Jurídicas.

Año V. Vol. V. №3. Edición Especial. 2020-III:

Universidad Regional Autónoma de los Andes

Hecho el depósito de Ley: FA2016000064

ISSN: 2542-3371

FUNDACIÓN KOINONIA (F.K). Santa Ana de Coro, Venezuela

Patty Del Pozo-Franco; Kevin Marcos Lynch-Becilla

Tabla 3.

Tabulación de encuestas a los estudiantes.

\begin{tabular}{|c|c|c|c|c|c|c|}
\hline $\mathbf{N}^{\circ}$ & ITEM & SI\% & NO\% & NULOS & $\begin{array}{c}\text { TOTAL } \\
\text { RESP. }\end{array}$ & $\%$ \\
\hline 1 & $\begin{array}{l}\text { ¿Los estudiantes deben aprender a } \\
\text { prevenir la violencia, para disminuir } \\
\text { conflictos escolares? }\end{array}$ & 97,3 & 2,7 & 1,8 & 486 & $98,2 \%$ \\
\hline 2 & $\begin{array}{l}\text { ¿En la actualidad algunos estudiantes } \\
\text { sancionados son reincidentes en } \\
\text { violencia, debido a que están } \\
\text { resentidos al ser sancionados por la } \\
\text { ley? }\end{array}$ & 72,3 & 27,7 & 3 & 480 & $97 \%$ \\
\hline 3 & $\begin{array}{l}\text { ¿Cree usted que los casos de violencia } \\
\text { por chismes en redes sociales, es } \\
\text { debido a que no se logra el Buen vivir? }\end{array}$ & 80,8 & 19,2 & 2,2 & 484 & $\mathbf{9 7 , 8 \%}$ \\
\hline 4 & $\begin{array}{l}\text { ¿Usted cree que se llama a la } \\
\text { POLICÍA, DINAPEN y GOE a la } \\
\text { salida de los estudiantes, es debido a } \\
\text { los diferentes tipos de conflictos que se } \\
\text { observan? }\end{array}$ & 76,2 & 23,8 & 2,2 & 484 & $\mathbf{9 7 , 8 \%}$ \\
\hline 5 & $\begin{array}{l}\text { ¿El reporte de inspectores sobre los } \\
\text { extraños en el plantel, se debe al } \\
\text { aumento de la violencia? }\end{array}$ & 64,1 & 35,9 & 4,4 & 473 & $95,6 \%$ \\
\hline 6 & $\begin{array}{l}\text { ¿Cree usted que la asistencia a talleres } \\
\text { de prevención de la violencia } \\
\text { organizados por el plantel, permite al } \\
\text { personal de la UEB ayudar en la } \\
\text { mediación? }\end{array}$ & 86,5 & 13,5 & 2,8 & 481 & $97,2 \%$ \\
\hline 7 & $\begin{array}{l}\text { ¿Considera que la disminución de } \\
\text { estudiantes sancionados, se debe a la } \\
\text { prevención del maltrato escolar? }\end{array}$ & 70,7 & 29,3 & 2,2 & 484 & $\mathbf{9 7 , 8 \%}$ \\
\hline 8 & $\begin{array}{l}\text { ¿Sabía usted que existen muchas } \\
\text { unidades educativas que no tienen } \\
\text { Centro de Mediación y solución de } \\
\text { conflictos, debido a que la ley no } \\
\text { obliga a crearlas? }\end{array}$ & 39,1 & 60,9 & 2,4 & 483 & $97,6 \%$ \\
\hline 9 & $\begin{array}{l}\text { ¿Por los docentes que forman } \\
\text { comisiones de control, ha disminuido } \\
\text { el índice de violencia? }\end{array}$ & 75,2 & 24,8 & 2,4 & 483 & $97,6 \%$ \\
\hline 10 & $\begin{array}{l}\text { ¿Las autoridades deben gestionar un } \\
\text { proyecto de creación obligatoria de un } \\
\text { centro de mediación y solución de } \\
\text { conflictos, debido a la necesidad de } \\
\text { prevenirlos? }\end{array}$ & 93,8 & 6,2 & 1,8 & 486 & $98,2 \%$ \\
\hline & TOTAL & $75,6 \%$ & $24,4 \%$ & $2,52 \%$ & 482,4 & $97,48 \%$ \\
\hline
\end{tabular}

Fuente: Resultados obtenidos del instrumento aplicado a la comunidad educativa Babahoyo. Autores (2020). 
lustitia Socialis. Revista Arbitrada de Ciencias Jurídicas.

Año V. Vol. V. №3. Edición Especial. 2020-III:

Universidad Regional Autónoma de los Andes

Hecho el depósito de Ley: FA2016000064

ISSN: 2542-3371

FUNDACIÓN KOINONIA (F.K). Santa Ana de Coro, Venezuela

Patty Del Pozo-Franco; Kevin Marcos Lynch-Becilla

Tabla 4.

Tabulación encuestas a personal administrativo.

\begin{tabular}{|c|c|c|c|c|c|c|}
\hline $\mathbf{N}^{\circ}$ & ITEM & SI\% & NO\% & NULOS & $\begin{array}{c}\text { TOTAL } \\
\text { RESP. }\end{array}$ & $\%$ \\
\hline 1 & $\begin{array}{l}\text { ¿El personal administrativo debe } \\
\text { aprender a observar los tipos de } \\
\text { violencia, para que disminuyan los } \\
\text { conflictos escolares? }\end{array}$ & 100 & 0 & 0 & 2 & 100 \\
\hline 2 & $\begin{array}{l}\text { ¿Cree usted que algunos estudiantes } \\
\text { sancionados son reincidentes en } \\
\text { violencia, debido a que están resentidos } \\
\text { al ser sancionados por la ley? }\end{array}$ & 0 & 100 & $\mathbf{0}$ & 2 & 100 \\
\hline 3 & $\begin{array}{l}\text { ¿Cree usted que los casos de violencia } \\
\text { por chismes en redes sociales, es debido } \\
\text { a que no se logra el Buen vivir? }\end{array}$ & 100 & 0 & 0 & 2 & 100 \\
\hline 4 & $\begin{array}{l}\text { ¿Usted cree que se llama a la POLICÍA, } \\
\text { DINAPEN y GOE a la salida de los } \\
\text { estudiantes, es debido a los diferentes } \\
\text { tipos de conflictos que se observan? }\end{array}$ & 100 & 0 & $\mathbf{0}$ & 2 & 100 \\
\hline 5 & $\begin{array}{l}\text { ¿El reporte de inspectores sobre personas } \\
\text { extrañas en el plantel, se debe al aumento } \\
\text { de la violencia? }\end{array}$ & 100 & 0 & 0 & 2 & 100 \\
\hline 6 & $\begin{array}{l}\text { ¿Cree usted que la asistencia a talleres de } \\
\text { prevención de la violencia organizados } \\
\text { por el plantel, permite al personal de la } \\
\text { UEB ayudar en la mediación? }\end{array}$ & 100 & 0 & $\mathbf{0}$ & 2 & 100 \\
\hline 7 & $\begin{array}{l}\text { ¿Considera que la disminución de } \\
\text { estudiantes sancionados, se debe a la } \\
\text { prevención del maltrato escolar? }\end{array}$ & 100 & 0 & $\mathbf{0}$ & 2 & 100 \\
\hline 8 & $\begin{array}{l}\text { ¿Existen muchas unidades educativas } \\
\text { que no tienen Centro de Mediación y } \\
\text { solución de conflictos, debido a que la } \\
\text { ley no obliga a crearlas? }\end{array}$ & 100 & 0 & 0 & 2 & 100 \\
\hline 9 & $\begin{array}{l}\text { ¿Por los docentes que forman comisiones } \\
\text { de control, ha disminuido el índice de } \\
\text { violencia? }\end{array}$ & 100 & 0 & 0 & 2 & 100 \\
\hline 10 & $\begin{array}{l}\text { ¿Las autoridades deben gestionar un } \\
\text { proyecto de creación de un centro de } \\
\text { mediación y solución de conflictos, } \\
\text { debido a la necesidad de prevenir antes } \\
\text { que sancionar? }\end{array}$ & 100 & 0 & 0 & 2 & 100 \\
\hline & TOTAL & $90 \%$ & $10 \%$ & $0,0 \%$ & 2 & $100 \%$ \\
\hline
\end{tabular}

Fuente: Resultados obtenidos del instrumento aplicado a la comunidad educativa Babahoyo. Autores (2020). 
lustitia Socialis. Revista Arbitrada de Ciencias Jurídicas.

Año V. Vol. V. №3. Edición Especial. 2020-III:

Universidad Regional Autónoma de los Andes

Hecho el depósito de Ley: FA2016000064

ISSN: 2542-3371

FUNDACIÓN KOINONIA (F.K). Santa Ana de Coro, Venezuela

Patty Del Pozo-Franco; Kevin Marcos Lynch-Becilla

\section{DISCUSIÓN}

Una vez obtenido los resultados se puede observar en cuanto a el interrogante número 10, la comunidad educativa considera necesario que las autoridades gestionen un proyecto para la creación de un centro de mediación y solución de conflictos, con el objeto de promover la prevención. Se manifestaron los docentes en un $86,4 \%$; los padres de familia $97.6 \%$; los estudiantes $93,8 \%$ y el personal administrativo un $100 \%$. En este sentido se puede mencionar lo plateado por (Merchán-Gailáñez, CadenaAlvarado \& Napa-Yance, 2019):

Si uno de los fines de las instituciones educativas es la socialización, los conflictos y las diferencias entre las personas forman parte de nuestras relaciones sociales, el sistema educativo debe asumir que ese proceso de socialización incluya el hacer frente a los problemas de convivencia, y el aprender a resolver los conflictos, de forma pacífica y cooperativa. (p.400)

Por todo ello es recomendable tener presente otros factores que permitan la canalización de los conflictos mediante una mediación productiva y cooperativa, reduciendo las posibilidades de violencia, entre estos tenemos (UNICEF, 2019):

- Personal docente sensible e informado.

- Actitud receptiva, atenta y cariñosa frente a los niños, niñas o adolescentes, por parte de las personas adultas que laboran en la escuela.

- Comunicación fluida entre la escuela y la familia. El padre, la madre, representantes o responsables forman parte activa de la comunidad educativa.

- Relaciones armónicas en el seno familiar del niño, niña o adolescente.

- Habilidades del niño, niña o adolescente para expresarse y para el manejo asertivo de conflictos.

- Que el niño, niña o adolescente cuente con varios amigos y amigas.

- Participación del niño, niña o adolescente en actividades alternativas a la escuela: deportivas, recreativas, culturales, comunitarias, entre otras.

- Disponibilidad de personal e infraestructura, para el apoyo y atención a niños, niñas y adolescentes con discapacidad.

- Cultura escolar de respeto a la diferencia. 
En este análisis también se evidenció muchos estudiantes nuevos cumplen trabajo comunitario, debido a los conflictos escolares. Así mismo, algunos estudiantes sancionados son reincidentes en violencia, debido al resentimiento por la aplicación de la ley. Falta de prevención inducida por la Ley de Arbitraje y Mediación. La violencia por chismes en redes sociales, se debe a que no se logra el Buen vivir. Reporte de inspectores sobre personas extrañas al plantel, debido al aumento de la violencia. Hay talleres de prevención de la violencia organizados por el plantel. Hay personal de la Unidad Educativa Babahoyo preparado sobre mediación. Existe disminución en los datos estadísticos de estudiantes sancionados. Las unidades educativas en Babahoyo, no tienen Centro de Mediación y solución de conflictos. Existe una interpretación inconclusa del Artículo 52 de la Ley de Arbitraje y Mediación. Los docentes forman comisiones de control y se organizan para que disminuya el índice de violencia. Las autoridades educativas del Distrito no gestionan proyectos de creación obligatoria de un centro de mediación y solución de conflictos. Y En correspondencia con lo declarado el Art. 130.11 del Código Orgánico de la Función Judicial, se refiere a las facultades jurisdiccionales de los jueces y juezas, indica que en todos aquellos asuntos en los que esté reconocida la transacción y de considerarlo oportuno, pueden disponer de oficio que pase el proceso a una oficina judicial de mediación intraprocesal, a fin de gestionar un arreglo voluntario que ponga fin al conflicto. Por esto es muy importante la mediación para el dialogo y acuerdos favorables, como lo plantea Santeliz-Contreras (2016) la mediación es, en definitiva, un proceso de resolución de conflictos, un cauce procedimental, un proceso de diálogo (p.39).

\section{RECOMENDACIONES}

Se logró llegar a las siguientes recomendaciones de acuerdo a la información obtenida mediante encuestas y análisis documental.

Para la promoción de un ambiente favorable en la unidad educativa, se bebe divulgar una adecuada interpretación del alcance del Artículo 52 de la Ley de Arbitraje y Mediación, así como promover su modificación, sobre la negligencia en el caso de no 
aportar a la solución de conflictos en su plantel. Gestionar a través de la motivación para que los docentes participen de las comisiones de control y se organicen para que disminuya el índice de violencia. Establecer una comunicar estrecha con las autoridades educativas del Distrito sobre la gestión de proyectos de creación obligatoria de un centro de mediación y solución de conflictos; sus ventajas para la ciudad y la comunidad estudiantil. Organización de talleres de socialización de la mediación para la solución de conflictos escolares. Desarrollar estrategias en la comunidad educativa para el control de personas extrañas al plantel. Continuar con los talleres de prevención de la violencia organizados por el plantel. Desarrollar talleres continuos sobre mediación, al personal de la Unidad Educativa Babahoyo.

\section{FINANCIAMIENTO}

No monetario.

\section{AGRADECIMIENTO}

A la Universidad Regional Autónoma de Los Andes; por motivar el desarrollo de la Investigación.

\section{REFERENCIAS CONSULTADAS}

Asamblea Nacional Constituyente de la República del Ecuador, (2008). Constitución de la República del Ecuador. [Constitution of the Republic of Ecuador]. Montecristi. Registro Oficial 449 de 20-oct-2008. Recuperado de https://n9.cl/sia

Asamblea Nacional del Ecuador. (2009). Ley Reformatoria al Código de la Niñez y Adolescencia. [Reform Law to the Childhood and Adolescence Code] Quito: Asamblea Nacional del Ecuador. Obtenido de https://n9.cl/ul23i

COFJ. (2009). Código Orgánico de la Función Judicial. [Organic Code of Judicial Function]. Quito: Registro Oficial. Suplemento 544 de 09-mar.-2009

Córdova Mendoz, K. T., Ochoa Espinoza, A. M., \& Durán Ocampo, A. R. (2019). Algunas consideraciones sobre la mediación y arbitraje. [Some considerations on mediation and arbitration]. Revista Universidad y Sociedad, 11(4), 287-295. 
lustitia Socialis. Revista Arbitrada de Ciencias Jurídicas.

Año V. Vol. V. №3. Edición Especial. 2020-III:

Universidad Regional Autónoma de los Andes

Hecho el depósito de Ley: FA2016000064

ISSN: 2542-3371

FUNDACIÓN KOINONIA (F.K). Santa Ana de Coro, Venezuela

Patty Del Pozo-Franco; Kevin Marcos Lynch-Becilla

Ley de Arbitraje y Mediación. [Arbitration and Mediation Act]. Registro Oficial 417 de 14 de diciembre 2006.

Merchán Gavilánez, M. L., Cadena Alvarado, R., \& Napa Yance, C. (2019). La mediación de conflictos escolares. Incidencia en el desarrollo de la inteligencia emocional. [Mediation of school conflict. Incidence in the development of emotional intelligence] Revista Conrado, 15(69), 399-404.

Ministerio de Educación (2012). Acuerdo Ministerial N0431-12. [Ministerial Agreement No. 0431-12].

Riofrío, J. (2015). La selección del método en la investigación jurídica. [Selection of the method in legal research]. Educación y Derecho. 12 (2015).

Santeliz-Contreras, M. (2016) Medios alternos de solución de conflictos inmersos en la legislación laboral de Venezuela. [Alternative means of conflict resolution immersed in Venezuela's labour law]. IUSTITIA SOCIALIS, 1(1), 31-48.

UNICEF (2019). Protocolo para entender, prevenir y reducir la violencia entre pares en las instituciones educativas. [Protocol to Understand, Prevent and Reduce Peer Violence in Educational Institutions]. Recuperado de https://www.unicef.org

\footnotetext{
(C)2020 por los autores. Este artículo es de acceso abierto y distribuido según los términos y condiciones de la licencia Creative Commons Atribución-NoComercial-Compartirlgual 4.0 Internacional (CC BY-NC-SA 4.0) (https://creativecommons.org/licenses/by-nc-sa/4.0/).
} 